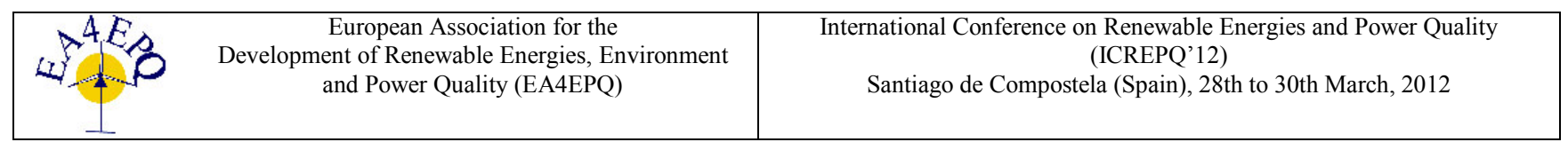

\title{
Accuracy Improvement in One-day Ahead Wind Power Output Prediction by Computational Fluid Dynamics Calculation
}

\author{
T. Kumano ${ }^{1}$ \\ ${ }^{1}$ Department of Electronics \& Bioinformatics \\ School of Science and Technology, Meiji University \\ 1-1-1 Higashi-mita, Tama-ku, Kawasaki, 214-8571 (Japan) \\ Phone/Fax number:+81 44 9347294, e-mail: kumano@isc.meiji.ac.jp
}

\section{Key words}

wind turbine generator, micro grid, output prediction, CFD (computational fluid dynamics), merit evaluation

\section{Introduction}

In the viewpoint of power system operation, accurate prediction of output from wind turbine generators becomes crucially important under the condition of large wind power penetration. The predicted wind power output is subtracted from the forecasted total demand and the fossil fuel powered generators are scheduled to be operated in the most economical way.

Because of the above stated importance, many works have been done concerning the wind speed forecast. Intelligent system methods such as fuzzy [1], ANN [2][3][4], time series analysis [5] have many examples of application for this purpose. On the other hand, CFD (Computational Fluid Dynamics) calculation has been long pointed out to be important in this field [6]. Some works discuss the importance of the spatial correlation and distribution of the prevailing wind [7], which implies the possibility that we can improve the accuracy of the wind speed prediction by fluid dynamics study.

The result of meso-scale meteorological prediction (weathre forecast) system by Japan Meteorological Agency (JMA) can be used via internet, and works have been done based on the utilization of this data as written in [8]. Because today's best expertise is there, it is the best tool for the analysis of the weather system dynamics. We can get highly likely prediction using this result. The only shortcoming is that the prediction is done only for the grid points, but not for the specific location of the wind turbine installation. Because the spatial wind distribution is affected by the terrain, in which the ground undulation of the size smaller than several hundred meters can give a considerable effects, but the mesh used in MSM (Meso
Scale Model) is as large as $5 \mathrm{~km}$ and the minimum spatial resolution of the used data of ground undulation is $1 \mathrm{~km}$.

Therefore, a detailed CFD analysis which takes into account of the local ground undulation using small mesh model is expected to improve prediction for the wind speed at a certain specific location. Ref [9] also describes that the meteorological data based CFD calculation is a strong tool for wind speed prediction. However, as far as the author knows, almost no works are published in the power system field so far concerning the accuracy of such calculation.

The objective of this paper is to show how we can improve the accuracy of the wind speed prediction by using the above stated CFD calculation procedure. Two 3D models of local ground surface are used, and the wind speed distribution is calculated using the GPV (Grid Point Value) data as the boundary condition. Attention is paid to the effect of model selection upon the prediction accuracy. Also shown is the merit of the prediction improvement by its effects on the system operation in the sense of the total fuel cost of the diesel generators in a micro grid.

\section{Proposed Method}

\section{A. GPV}

JMA delivers MSM-GPV data every 6 hours for all the $5 \mathrm{~km}$ grid points on and around Japan Islands. The available data are wind speed and direction, precipitation, atmospheric pressure, etc.. Prediction in MSM-GPV starts from $t_{s}=0,6,12,18$ hour and gives hourly values until $t=t_{s}+33 \mathrm{~h}$. These data can be opened via web with several minutes delay. In the proposed system, the GPV prediction starting from 6 am is used and the target time range is 24 hours starting from 9 am. Three hours are given to enable us to execute preliminary process needed for the power system daily operation. 
The data given to the nearest grid point is used for the input data in the CFD analysis. Because the GPV prediction is done hourly, the output prediction by the proposed system is also given once every hour. On the other hand, 10 minutes sampling wind speed by AMeDAS (Automated Meteorological Data Acquisition System) are averaged hourly and treated as the errorless measurement.

\section{B. CFD Computation}

Wind Perfect DX (WPDX) by Environment Simulation Inc. is used for CFD calculation. It automatically checks the numerical stability and switches the integration scheme. The GPV wind speed data is given as the boundary condition to the four rectangular side planes and the top horizontal plane. The vertical component of wind speed is neglected in the boundary condition. Automatic meshing mechanism of WPDX is used.

Basically the calculation is numerical integration of Navier-Stokes equation, which is nonlinear, and needs thorough analysis varying wind speed and direction. In the example here, the point of wind turbine installation is Point A in Fig. 1. Therefore, the author conducted wind speed field calculation for 64 cases, in which 3, 6, 9, and $12 \mathrm{~m} / \mathrm{s}$ are assumed as wind speed and 16 directions from north to north by north west are assumed. The result of this calculation is summarized by a radar chart as in Fig. 2 . Once the predicted value of wind speed and direction is obtained by GPV, this radar chart will give us the predicted wind speed at the specific point of the wind mill. Because this chart maps the input GPV wind prediction to the output actual wind at the particular studied location considering the terrain around, it is called terrain map hereafter for convenience.

In the present paper, two terrain models are used; one is $1 \mathrm{~km}$ by $1 \mathrm{~km}$, and the other is $2 \mathrm{~km}$ by $2 \mathrm{~km}$. Without question, the former takes shorter computation time but the latter is more accurate, in which the aerodynamic effect of the neighboring terrain is also considered.

\section{Output Power Prediction}

AMeDAS measures the wind speed at the height of $15.1 \mathrm{~m}$ above ground in the case studied here, but the wind mill is usually placed higher. In this case the height of the windmill is assumed to be $45 \mathrm{~m}$. Its major specifications are summarized in Table I. Logarithmic function is assumed as the vertical distribution of wind speed as shown in Eq. (1).

$$
v_{w}=v_{w o}\left(\frac{h}{h_{o}}\right)^{n}
$$

where $v_{w}$ and $v_{w o}$ stand for wind speeds at field point and its nominal observation height, respectively. The nominal wind speed is that at the height of the nominal observation height $h_{o} . h$ is the height of field point. $n(=0.15)$ is a shape coefficient. Effective wind speed for estimating wind power output can be easily obtained by this equation.
Table I. - Major specifications of the wind power station.

\begin{tabular}{|l|c|}
\hline \multicolumn{1}{|c|}{ Items } & Assumed Data \\
\hline Rated output power & $600 \mathrm{~kW}$ \\
\hline Tower height & $45 \mathrm{~m}$ \\
\hline Wind mill Rotor diameter & $48 \mathrm{~m}$ \\
\hline Cut-in wind speed & $3 \mathrm{~m} / \mathrm{s}$ \\
\hline Rated wind speed & $13 \mathrm{~m} / \mathrm{s}$ \\
\hline Cut-out wind speed & $20 \mathrm{~m} / \mathrm{s}$ \\
\hline
\end{tabular}

So called power curve is used to give electrical power output from the input of the wind speed. The concrete example of the used power curve is later shown in Fig. 4. The output power wave might have a different shape from that of the wind speed because the power curve has a strong nonlinearity.

\section{Merit Evaluation}

For the purpose of demonstrating the merit of the prediction accuracy in the viewpoint of power system operation, the total fuel cost of the diesel generators necessary to feed demand is calculated for the two cases;

1) Case 1: The smaller terrain model $(1 \mathrm{~km} \mathrm{X}$ $1 \mathrm{~km}$ ) is used in CFD analysis

2) Case 2: The larger terrain model $(2 \mathrm{~km} \mathrm{X} 2 \mathrm{~km})$ is used.

In both cases the load forecast is assumed to be perfect (no prediction error). Because the actual wind speed is different from the prediction, the actual electrical power available from the wind turbine generator is also different from the predicted value. Therefore we have certain amount of power mismatch between the necessary diesel output and the total of the scheduled output. This mismatch is supplied by BESS (Battery Energy Storage System), which we assume to be readily installed to the wind power generator, and its energy capacity enables the rated output power for as long as 1 hour. Up to the upper and lower limits of the stored energy of the battery, the diesel generators will give the same output as scheduled which is the result of the pre-processed optimization, but the fuel cost makes difference beyond the limit of the battery storage. The outline of the assumed micro grid is shown in Table II (next page). It is easily expected that the more accurate the wind power output prediction, the smaller the fuel cost.

\section{Numerical Example}

\section{A. Studied Region and Terrain}

The usefulness of the proposed method is verified numerically using the actual wind speed measurement. The studied wind turbine generator is assumed to be located at one of the AMeDAS observation point. (Point A in Fig. 1) AMeDAS observes meteorological data such as wind speed and direction, amount of solar radiation, and precipitation, and so on. It automatically measures these data by 10 minutes sampling and published every next day. 
Table II. - Outline of the studied micro grid.

\begin{tabular}{|l|c|}
\hline \multicolumn{1}{|c|}{ Items } & Assumed Data \\
\hline Total generating capacity & $3.1 \mathrm{MW}$ \\
\hline Rated output of Gen. 1 & $1.5 \mathrm{MW}$ \\
\hline Speed regulation Gen 1 & $3 \%$ \\
\hline Rated output of Gen. 2 & $1 \mathrm{MW}$ \\
\hline Speed regulation Gen2 & $4 \%$ \\
\hline
\end{tabular}

* including WTG in Table I.

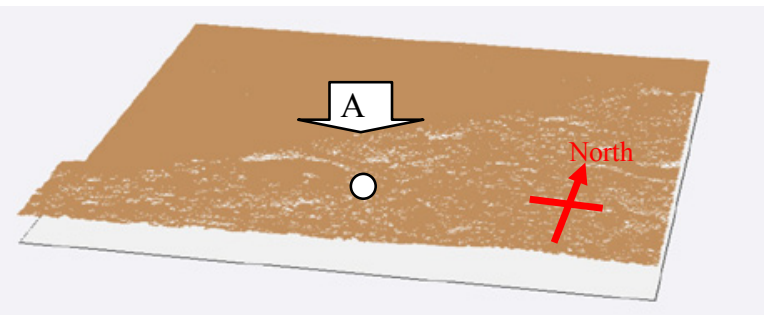

(a) $1 \mathrm{~km} \mathrm{X} 1 \mathrm{~km}$ region

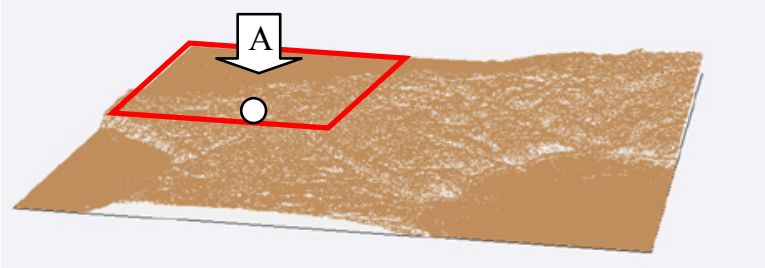

(b) $2 \mathrm{~km} \mathrm{X} 2 \mathrm{~km}$ region (the red parallelogram is (a))

Fig. 1. Studied location and terrain

The terrain around Point $\mathrm{A}$ is as shown in Fig. 1. Point $\mathrm{A}$ is located in a cape out into the pacific ocean (south) from the main island (north), and is just at the top of the hill aside the north west coast of the cape. On the contrary, the east and south back of Point $\mathrm{A}$ is mountainous regions. Even if the GPV predicted high wind speed, the actually observed wind speed at Point A might be far smaller particularly in case of eastern and southern wind because of the barrier effect of these mountains.

\section{B. Terrain Mapping}

The terrain mappings obtained from CFD computation are shown in Fig. 2. In each radar chart, four curves are given which are the expected wind speed at the observation point (Point A) in cases of 3, 6, 9, and $12 \mathrm{~m} / \mathrm{s}$ cases. The directional characteristics stated in the previous subsection can be clearly observed. Once this terrain mapping is obtained, it can be used for any GPV prediction. For this wind speed in between these values, linear interpolation is used.

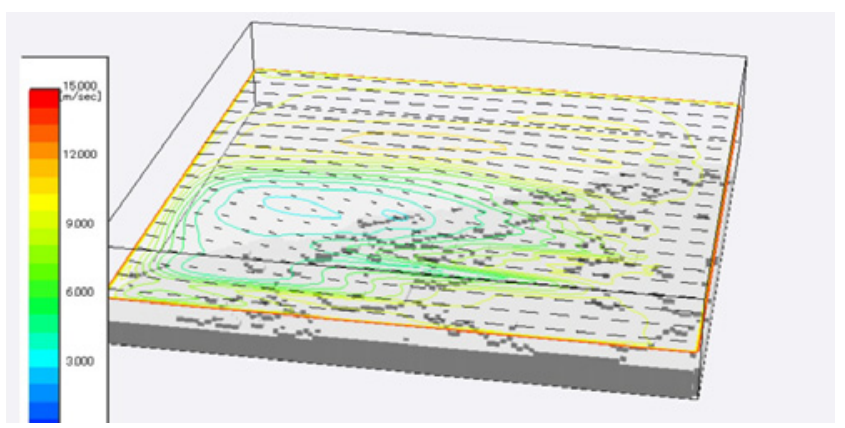

(a) Case 1
Fig. 3 shows examples of CFD computation results. The coloured plane is located at $83 \mathrm{~m}$ above the sea level, which is approximately at the same elevation as AMeDAS measurement. In Case 1 most of the inlet wind from the eastern boundary is obstructed by the high mountains, considerably weakening the wind. In Case 2 the effect of relatively large open space (sea region) around the southeastmost corner is taken into account, and no big mountain blocking effect is observed.

\section{Wind Speed and Wind Power Output}

The proposed method is tested using the data measured for seven consecutive days. Fig. 5 shows the result. The GPV forecast and the measured wind directions are compared. (Fig. 5(a)) On the other hand, Figs. 5(b) and 5(c) show the wind speed and the wind turbine generator output respectively. The three charts here are the measured data, the predicted data in Cases 1 and 2 . Owing to the high accuracy in GPV data, it is easily observed that the proposed method gives a good prediction. Compared between the two cases, Case 2 (using the larger terrain) is more accurate. Fig. 4 gives the used power curve of the wind power generator, as already mentioned in Section C of Chapter 2.

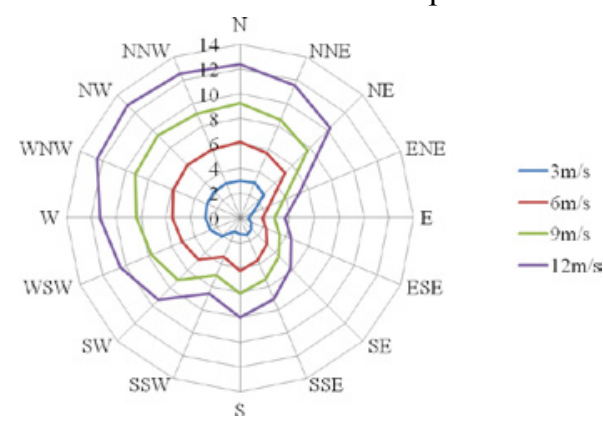

(a) Case 1

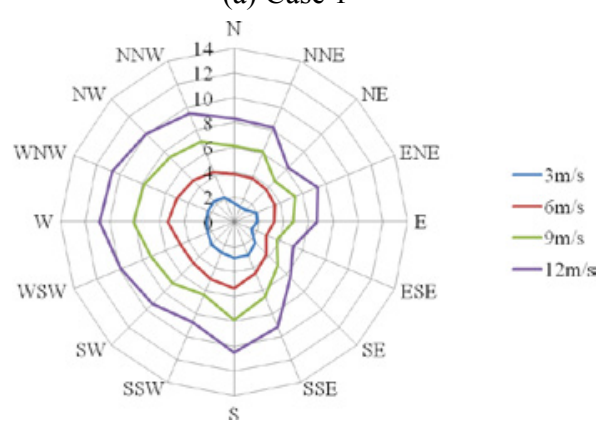

(b) Case 2

Fig. 2. Terrain Mappings

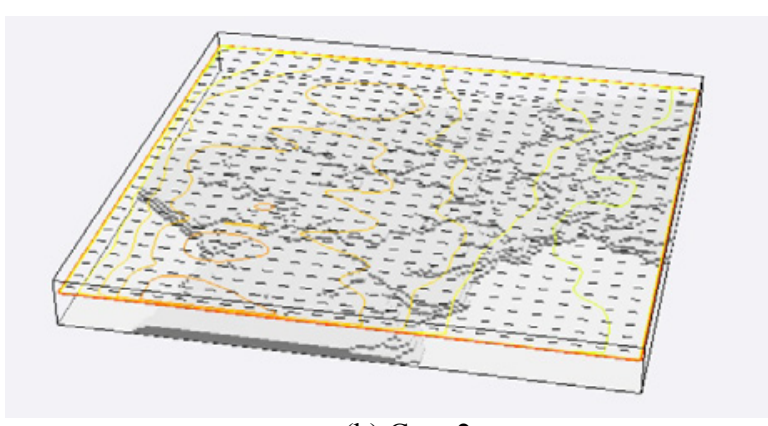

(b) Case 2

Fig. 3. Examples of CFD calculation 


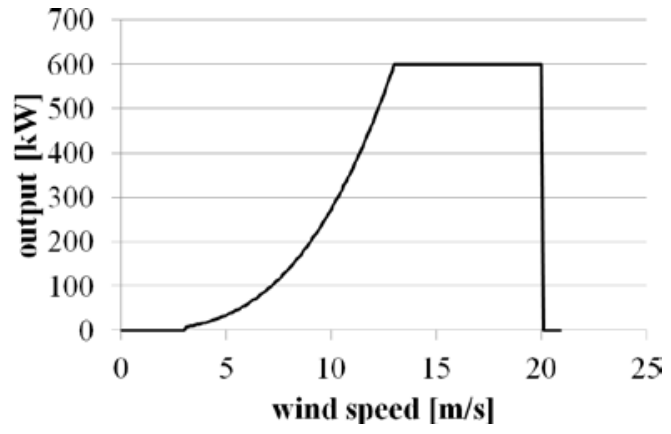

Fig. 4. Power curve

- GPV - measured

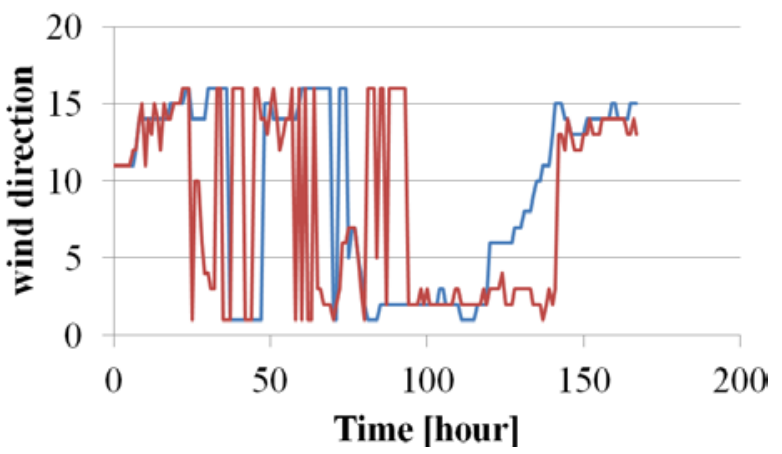

(a) wind direction

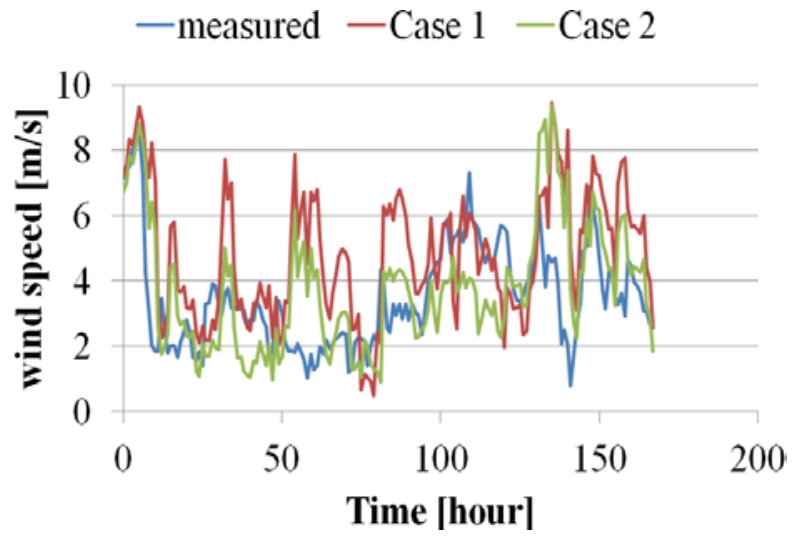

(b)wind speed

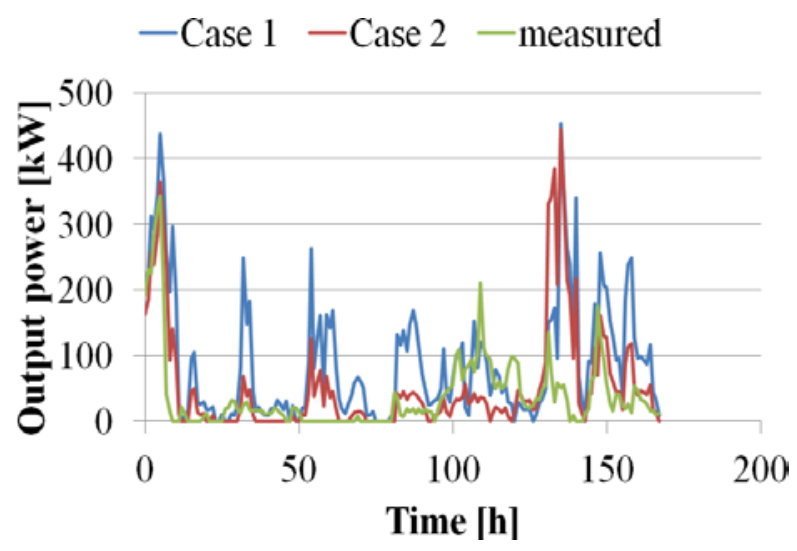

(c)wind turbine generator output

Fig. 5. Prediction results

In Fig. 5(c), because of the nonlinearity inherent in the power curve, the discrepancy between the prediction and the actual becomes larger than that of wind speed. Again, Case 2 gives more accurate prediction.

\section{Merit Evaluation}

Based on the load variation scenario shown in Fig. 6(a), daily operation pattern for the diesel generators is determined in Cases 1 and 2 as shown in (b) and (c). Here the area between the red and blue curves stands for the output from DG2, and that below the blue curve DG1. The load demand assumed here is based on the weekly load curves of several utility companies which is opened in the web. The initial state of charge in BESS is assumed to be $50 \%$. Because of the difference between the load and the scheduled diesel output, output command is sent to the battery as shown in Fig. 6(d). As far as BESS can supply this required power, diesels are not required to change its output from the schedule, whereas BESS varies its internal state. In Case 1 the stored energy in BESS violates the lower limit for about $60 \%$ of the whole operating hours, but in Case 2 the situation is neutralized and for only $45 \%$ energy storage hit the limitation.

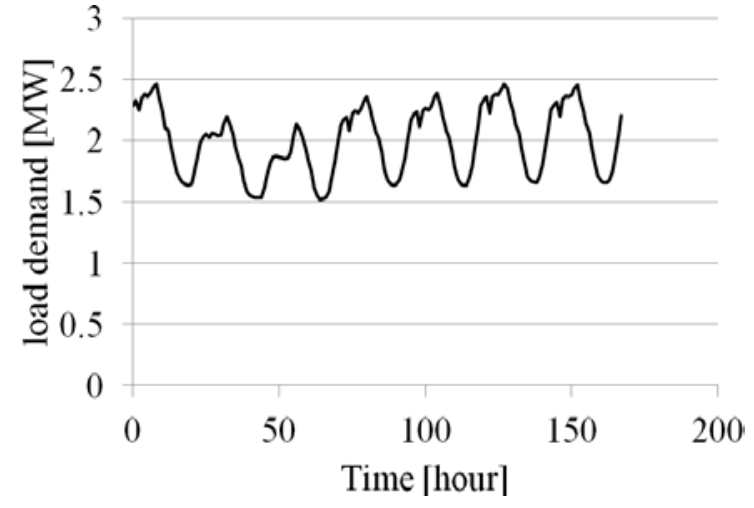

(a) load demand

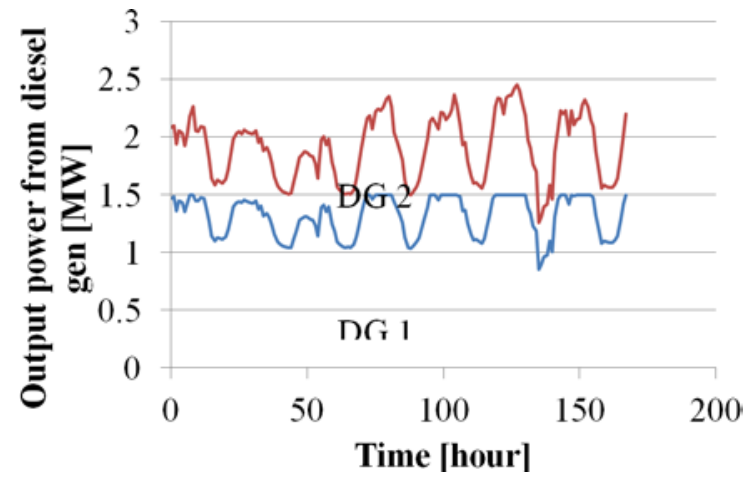

(b) Diesel generators operation shcedule in Case 1

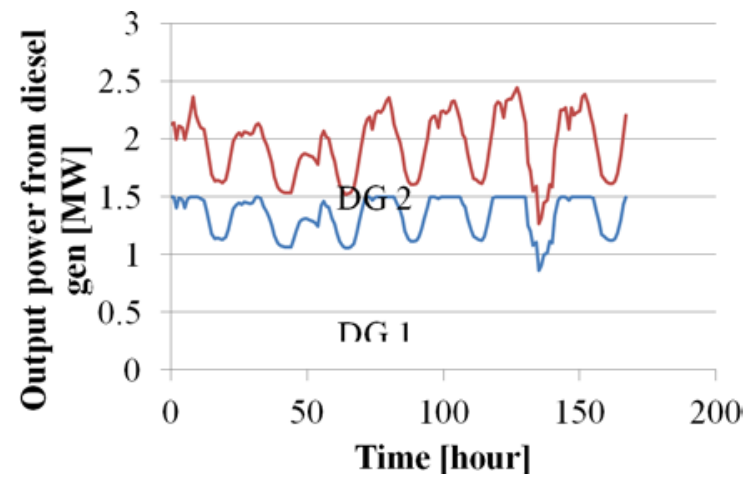

(c) Diesel generators operation shcedule in Case 2

Fig. 6. Merit evaluation (continued to next page) 


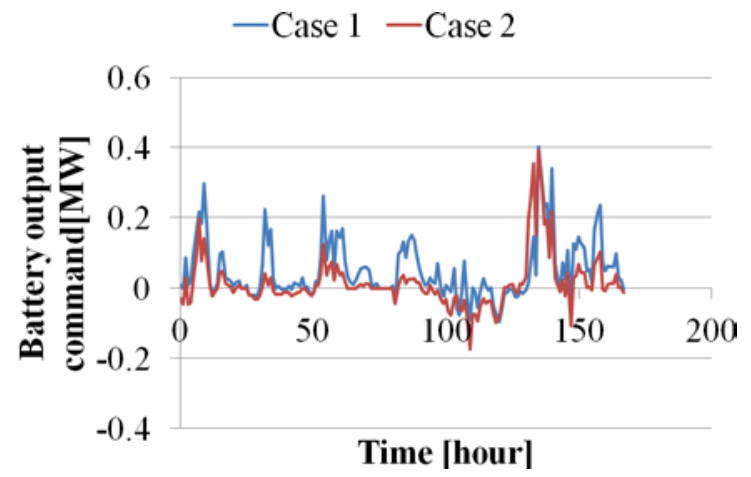

(d) output command sent to battery

- Case 1 - Case 2

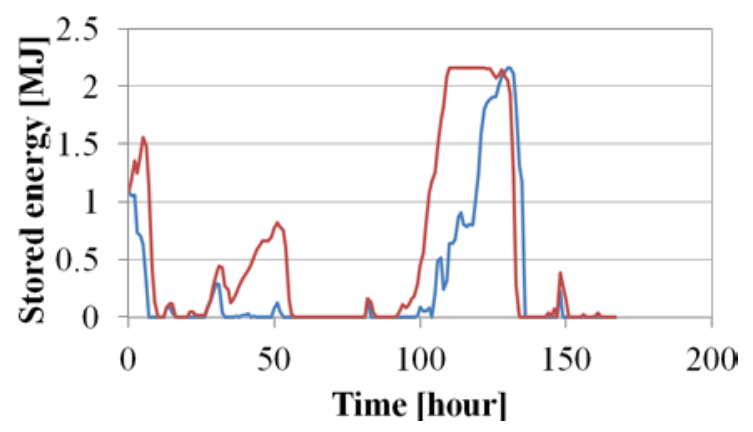

(e) stored energy

Fig. 6. Merit evaluation

Table III. - Total fuel cost

\begin{tabular}{|l|c|}
\hline & Total cost [pu] \\
\hline Case 1 & 394.74 \\
\hline Case 2 & 394.70 \\
\hline
\end{tabular}

In the case of the violation of BESS stored energy, the power mismatch is compensated by both diesels. Because the speed regulation in DG1 is smaller than in DG2, its output is required to be adjusted more than DG2. The output capacity of DG1 and DG2 is $1.5 \mathrm{MW}$ and $1 \mathrm{MW}$ respectively, and DG2 is sometimes forced to increase its output because of the upper limit of DG1. The overall fuel cost is calculated as shown in Table III. It is confirmed that Case 2 is better in the sense of economy, which implies that it consumes less fuel emitting less $\mathrm{CO}_{2}$. The bigger expected cost in Case 1 results from the too much violation of the battery storage energy due to the incorrect wind output power prediction.

\section{Conclusions}

This paper proposes a method to apply CFD calculation to improve the one day-ahead wind power output prediction By CFD calculation we can take into account the effect of the local terrain upon the wind speed at the particular location of wind power plant installation. The terrain mapping, which gives the local wind speed expected from MSM-GPV prediction, is proposed to be used. Two CFD calculation models are used and compared, which in turn give two terrain mappings respectively. A numerical example is used to demonstrate the effectiveness of the proposed method. It is shown that the local terrain model used in CFD analysis can give a significant difference in the terrain mapping and the accuracy of the output prediction. The larger CFD model is better in the sense of prediction, which simulates the aerodynamic effects of the local terrain more accurately.

The merit of the prediction accuracy is quantitatively evaluated in the sense of power system operation showing the necessary fuel cost impact. The terrain mapping introduced in the present paper is intuitively easy to understand and the author believes that it can become a powerful tool for more accurate wind speed and output power prediction.

\section{Acknowledgement}

This work was supported by JSPS/MEXT Grant-in-Aid for Scientific Research (C) KAKENHI (22560282).

\section{References}

[1] M. Gavrilas, G. Gavrilas, "An enhanced ANN wind power forecast model based on a fuzzy representation of wind direction", 10th Symposium on Neural Network Applications in Electrical Engineering, pp. 31-36, 2010

[2] K.Taniguchi, K.Ichiyanagi, K. Yukita, and Y. Goto, "Study on Forecast of Time Series of Wind Velocity for Wind Power Generation by Using Wide Meteorological Data", Trans IEEJ, Vol 128-B, No 2, pp416-422, 2008

[3] S. Salcedo Sanz, A. Pe'rez-Bellido, E. Ortiz-Garcia, A. Portilla-Figueras, L. Prieto, D. Paredes, and F. Correoso, "Short-term Wind Speed Prediction by Hybridizing Global and Mesoscale Forecasting Models with Articial Neural Networks", Proc. IEEE 8th International Conference on Hybrid Intelligent Systems, pp608-612, 2008

[4] H.M.I. Pousinho, V.M.F. Mendes, and J.P.S. Catalão, "Neuro-Fuzzy Approach to Forecast Wind Power in Portugal”, Proc of ICREPQ '10, Paper No 222 (2010)

[5] Chi-Yo Huang, Yu-Wei Liu, Wei-Chang Tzeng and Po-Yen Wang, "Short Term Wind Speed Predictions by Using the Grey Prediction Model Based Forecast Method", IEEE Green Technologies Conference (IEEE-Green), 2011, Digital Object Identifier: 10.1109/GREEN.2011.5754856

[6] A. M. Foley, "Wind Power Forecasting \& Prediction Methods", Proc IEEE 9th Int. Conf. on Environment and Electrical Engineering., Prague, Czech, May 16-19, (2010)

[7] I. Damousis, et al, "A fuzzy model for wind speed prediction and power generation in wind parks using spatial correlation", IEEE Trans on Energy Conversion, Volume: 19 , No: 2 2004, pp352 - 361

[8] T. Tanabe,et al, "Generation scheduling for wind power generation by storage battery system and meteorological forecast", Power and Energy Society General Meeting Conversion and Delivery of Electrical Energy in the 21st Century, 2008 IEEE, Digital Object Identifier: 10.1109/PES.2008.4596116

[9] A. Szekely, T. Barbarics, "Short-term prediction of the power generation of wind turbines", Proc of ICREPQ '06, Paper No 295 (2006) 